\title{
Morphology Study of Particle Breakage Mechanisms in a Horizontal Stirred Mill: Automated and Manual Point Counting Approaches
}

\author{
Reem Roufail1, Bern Klein² \\ ${ }^{1}$ Systems Design Engineering, University of Waterloo, Waterloo, Canada \\ ${ }^{2}$ Norman B. Keevil Institute of Mining Engineering, University of British Columbia, Vancouver, Canada \\ Email: rroufail@uwaterloo.ca, bklein@mining.ubc.ca
}

How to cite this paper: Roufail, R. and Klein, B. (2021) Morphology Study of Particle Breakage Mechanisms in a Horizontal Stirred Mill: Automated and Manual Point Counting Approaches. Journal of Minerals and Materials Characterization and Engineering, 9, 528-543.

https://doi.org/10.4236/jmmce.2021.95035

Received: August 5, 2021

Accepted: September 27, 2021

Published: September 30, 2021

Copyright $\odot 2021$ by author(s) and Scientific Research Publishing Inc. This work is licensed under the Creative Commons Attribution International License (CC BY 4.0).

http://creativecommons.org/licenses/by/4.0/

\begin{abstract}
High-speed stirred mills are utilized to grind particles below $10 \mathrm{~mm}$. Grinding sulphide minerals to as low as $10 \mathrm{~mm}$ achieve adequate mineral liberation for successful downstream mineral processing operations, such as flotation and leaching. Particle breakage mechanism such as fracture or abrasion, determines the morphological surface features of the product particles. It is anticipated that particles, which break along grain boundaries (intergranular) produce rough surfaces, whereas particles that break across the grain boundaries (transgranular) possess smoother surfaces. In this study, particles are ground in a stirred mill and their morphological features were analyzed using automated and manual detection methods. Literature and conventional belief are that high-speed stirred mills break particles by attrition. This paper showed that fracture is also an important breakage mechanism along with attrition. Breakage mechanism is a factor of input stress intensity, in the form of the mill agitator speed, and type of mineral. It is observed that at higher agitator speed galena fractures along the grain boundaries, whereas quartz, abrade across the grain boundaries.
\end{abstract}

\section{Keywords}

Comminution, Morphology, Breakage Mechanism, Stirred Mill, Grinding, Manual Point Counting

\section{Introduction}

The increase of mineralogical complexity of ore bodies lead to the investigation 
of different tools that can increase liberation which would enhance the recovery grade of sulfide minerals. A few ore pretreatment technologies have been studied, for example, microwave, ultrasonic, bio-milling, shock wave and others. Such technologies are showing promising results, however, they are expensive to adopt and energy-intensive [1]. One of the solutions that is currently employed by the mining industry is grinding the particles to $10 \mu \mathrm{m}$ or less via high-speed stirred mills.

The highest energy consumption in a mining operation is consumed by grinding processes, to achieve fine ground products that are reasonably liberated. Therefore, research studies are focused on fine grinding operations, such as stirred mills. Becker, Kwade, and Schwedes [2], studied the relationship of different forms of energy to stress intensity and final product particle size. Others studied mill design and stress intensity distribution [3] [4] [5] [6]. Peukert [7] studied the effect of the mechanical properties of the grinding media and ground material on the comminution process. Bu et al. [8] studied the effect of wet versus dry grinding on particle morphology features and their effect on the flotation process. Moosakazemia et al. [9] investigated the effect of ball mill liner and operating conditions, such as quartz feed size and its morphology features.

Promoting breakage mechanism that would achieve mineral liberation at the lowest energy consumption is imperative to conserve comminution energy consumption. Breakage mechanism that promotes intergranular breakage over transgranular would achieve a high level of liberation at the coarser ground product. According to Tromans and Meech [10] and Celik and Oner [11], intergranular fracture requires $10 \%-14 \%$ less energy than transgranular. Promoting intergranular breakage by adjusting operation conditions based on types of minerals in the mill is the least risk-taking adjustment to the industry. Building up the knowledge of particle breakage features of different types of mineral rocks based on mill operating conditions can lead to an informative mill operation beyond particle size reduction and distribution.

The focus of this study is to understand the effect of different mill operating parameters and types of minerals on particle breakage mechanisms. Operating parameters are summarized as stress intensity applied on the particles and different mineral physical properties. Two mineral types are selected for the study, which are hard/low-density minerals (quartz) versus soft/high-density minerals (galena). According to Blecher [5], stress intensity in stirred mill is a function of the density and size of the grinding media and the agitator tip speed. In this study, agitator speed is studied as a stress intensity control and the density of the ground mineral is addressed.

Morphology is utilized in this study to evaluate the breakage mechanism of mineral particles. In literature, morphology is addressed both qualitatively and quantitatively. Qualitative analysis is a visual comparison of captured high-resolution images via SEM, TEM and X-ray spectroscopy [12] [13] [14], others performed quantitative analysis and measured morphological parameters, such as elongation, sphericity, and roughness, media wear [3] [12] [15] [16]. Morpholo- 
gy analysis in this study is quantitative, where an automated method using Clemex software [17], and a manual point counting method were employed [18].

\section{Experimental Work}

\subsection{Material and Experimental Procedures}

The minerals chosen for the study were galena concentrate and quartz, because of their distinct physical properties. Galena has a distinct cleavage, compared to quartz. Galena has an octahedral crystal structure with a distinct cleavage of cubic structure on [001] and parting on [111] and it fractures with Subconchoidal feature, compared to quartz that has a trigonal crystal structure with an indistinct cleavage $\{0110\}$ and Conchoidal fracture. However, mineral selection for this study was not based on cleavage and crystal structure. The focus was on physical properties of the minerals, hardness, and density. Mohs' hardness value for galena is about 2.5 (very soft) while quartz is close to 7.0 (very hard). Galena is a heavy mineral that has a measured SG of 7.19, compared to the quartz, which has a measured SG of 2.63. Quartz and galena were studied separately. Then a mixture of both minerals was synthesized to simulate ore composition with different types and properties of minerals. The synthesized mixture of galena and quartz were prepared with a ratio of 1 to 6 by volume. Particle sizes of feed minerals (P80) were not too different, where quartz, galena and mixture were 97.4, 96.6 and $122.8 \mu \mathrm{m}$, respectively. The particle size distribution of galena sample is wider than quartz, Rosin Rammler distribution modulus was 4.42, 1.34 and 1.46 for quartz, galena, and mixture sample, respectively. Feed particle size distributions for quartz, galena and mixture sample are presented by the solid line in Figures 1-3, respectively.

The three types of samples, quartz concentrate, galena concentrate, and mixture sample of quartz and galena were exposed to grinding in 4-litre Netzsch IsaMill (LME4) by Xstrata Technology. Due to the wide range of densities of the tested minerals and to achieve a pulp density of about $45 \%$, the percent solids were fixed at $14.3 \%$ by volume. The solid concentration by mass for quartz was $30.5 \%$, galena concentrate was $54.4 \%$ and mixed quartz and galena was $36 \%$. The grinding media used was a mono size ceramic bead, (f $2 \mathrm{~mm}$ ). The media filled $80 \%$ the effective mill volume.

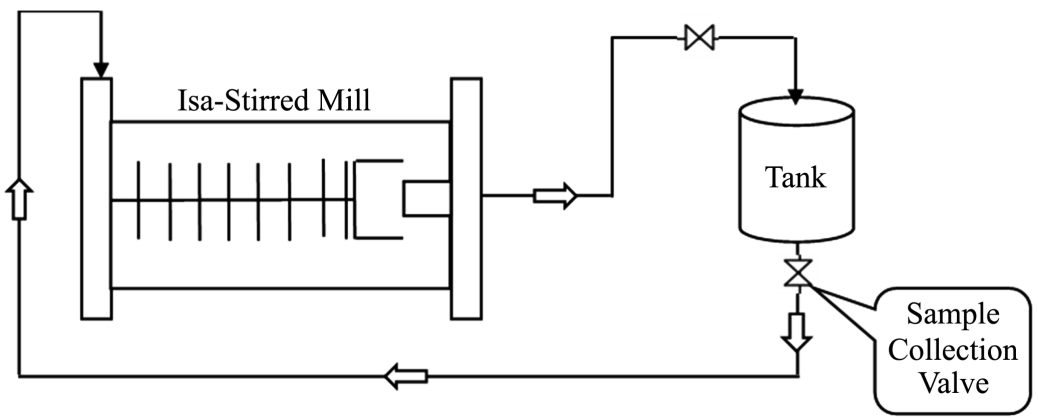

Figure 1. Schematic diagram of experimental flow. 

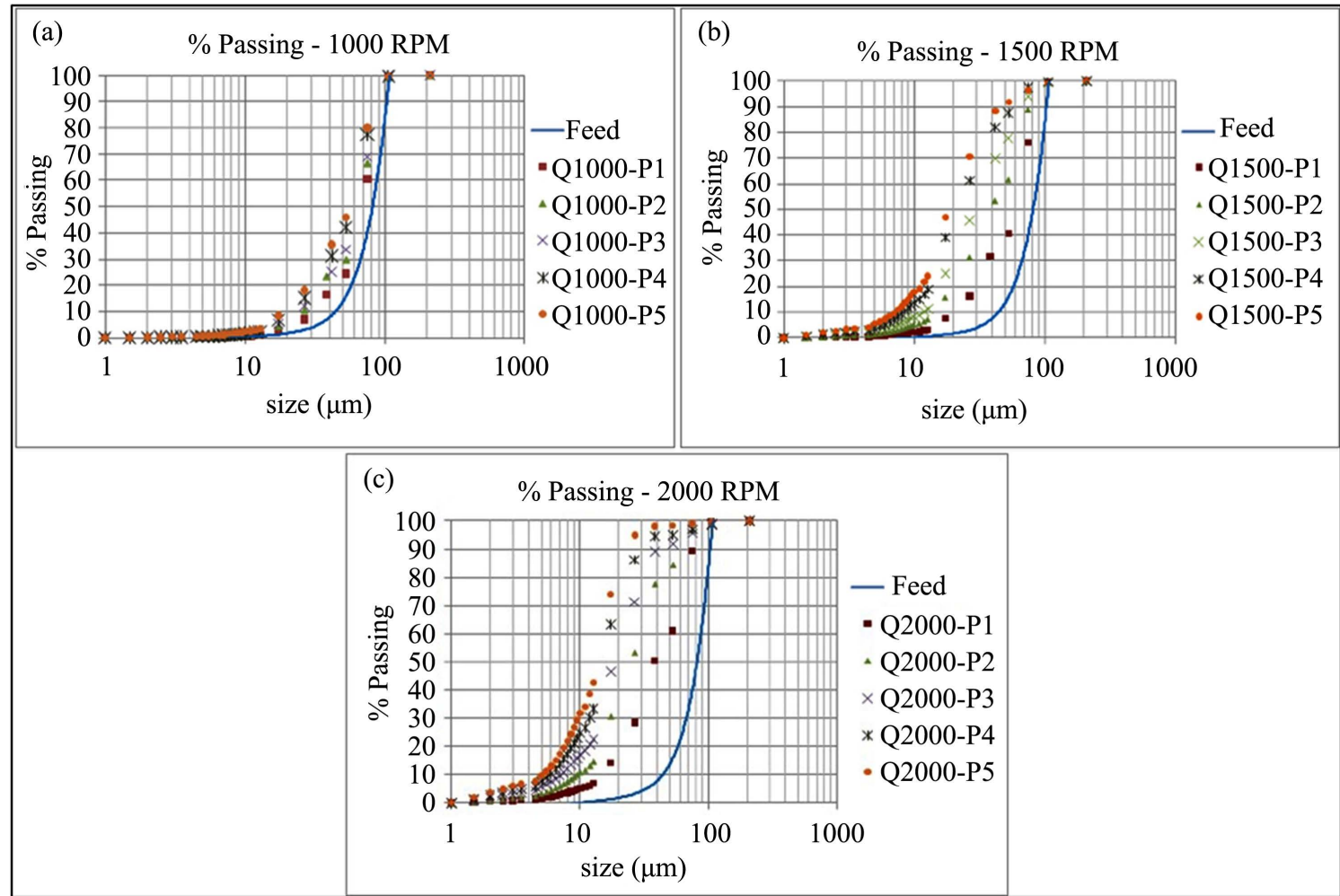

Figure 2. Quartz passing percent for (a) 1000, (b) 1500 and (c) $2000 \mathrm{rpm}$.

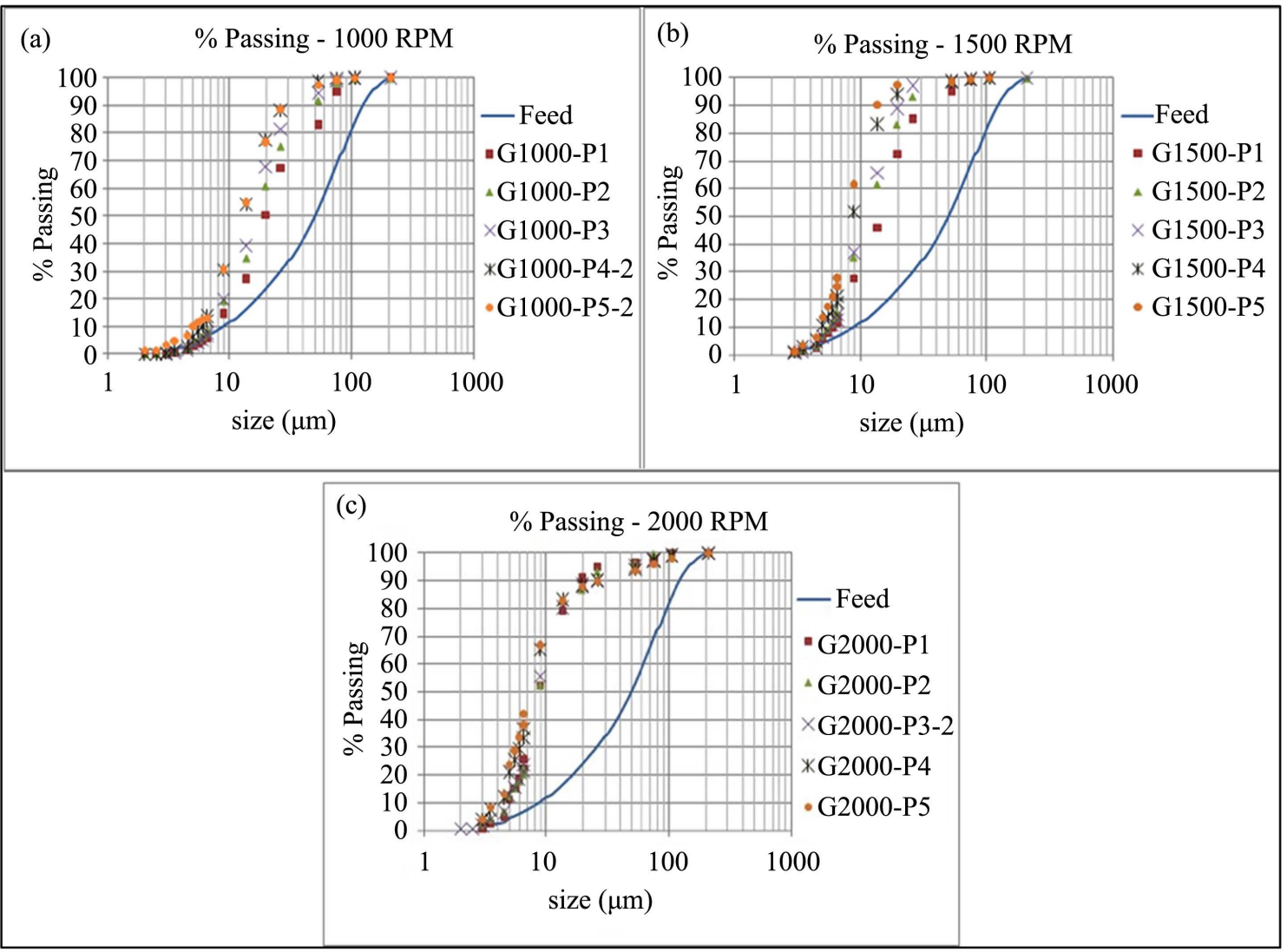

Figure 3. Galena concentrates passing percent for (a) 1000, (b) 1500 and (c) $2000 \mathrm{rpm}$. 
Three agitator speeds, which will affect stress intensity levels in the mill, were examined for each mineral, 1000, 1500 and $2000 \mathrm{rpm}$. The pulp flow rate was set at the highest setting that the machine can handle, $3.5 \mathrm{~L} / \mathrm{min}$. The highest flow rate is applied to achieve the lowest residence time of the particles in the mill per pass. The effect of residence time and the secondary breakage of the particles were studied by running the same mineral composition through the mill 5 times (5 passes), as illustrated in Figure 1.

\subsection{Particle Size Distribution}

Particle size distribution for quartz, galena, and mixed sample at the three agitator speeds and feed are shown in Figures 2-4, respectively. It is observed that grinding limit is a factor of energy input and mineral type. Grinding limit is the residence time and energy input if reached, no further particle size reduction will occur.

Quartz reached its grinding limit at low agitator speed (1000 rpm) with size reduction of only $23 \%$ after first pass. Galena on the other hand reached its grinding limit at highest agitator speed $(2000 \mathrm{rpm})$ with $84 \%$ size reduction after first pass, as shown in Table 1. Size reduction of mixed sample had similar features as pure quartz sample at low agitator speed. Mill input energy in the form of agitator speed affects particles breakage behavior, which is observed by size distribution and size reduction analysis, which is in agreement with Parry's findings [19].

Table 1. Particle size reduction.

\begin{tabular}{ccccccc}
\hline & \multicolumn{5}{c}{ Particle Size Reduction \% } \\
\cline { 2 - 6 } $\begin{array}{c}\text { Grinding Speed } \\
(\text { RPM })\end{array}$ & \multicolumn{2}{c}{ Quartz } & Galena & Mixed \\
& \multicolumn{2}{c}{ (Quartz + Galena) } \\
\cline { 2 - 6 } & $23 \%$ & $23 \%$ & $48 \%$ & $79 \%$ & $19 \%$ & $19 \%$ \\
1000 & $23 \%$ & $69 \%$ & $76 \%$ & $86 \%$ & - & - \\
1500 & $28 \%$ & $79 \%$ & $84 \%$ & $84 \%$ & $39 \%$ & $85 \%$ \\
\hline
\end{tabular}

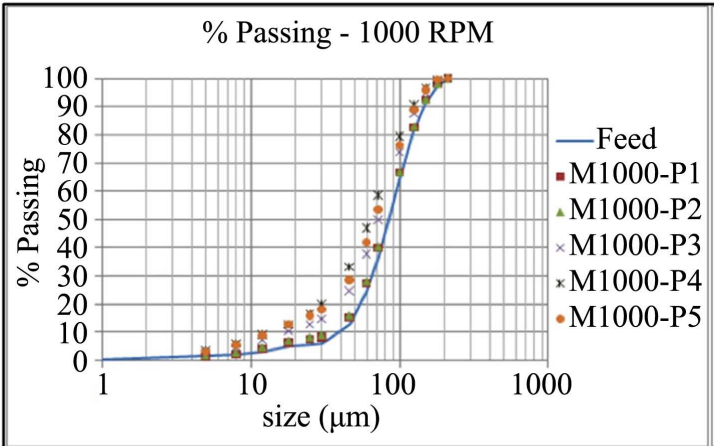

(a)

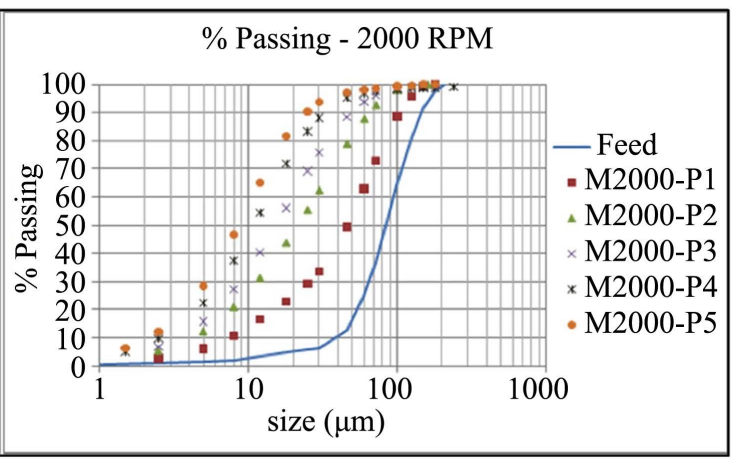

(b)

Figure 4. Mixed quartz and galena sample passing percent for (a) 1000 and (b) $2000 \mathrm{rpm}$. 
Three size fractions were used for morphology analyses. They are referred to as coarse, medium, and fine. The coarse fraction geometric mean size was $63 \mu \mathrm{m}$, which is prepared by dry screening. The medium and fine fractions were produced using Cyclosizer, which generated products with varying particle sizes based on the mineral density. Therefore, the size fraction for each mineral was chosen based on the particle size, rather than the Cyclosizer cyclone number. The geometric mean sizes for quartz, galena concentrate, and mixed quartz and galena samples were 34, 37 and $36 \mu \mathrm{m}$ for the medium size fraction and 15, 17 and 18 $\mu \mathrm{m}$ for the fine size fraction, respectively.

\subsection{Morphology Analysis}

In this study morphology is analyzed using two methods for validation; an automated method (Clemex) that identifies the basic features such as, sphericity, elongation and roughness, and a manual point counting method that recognize similar features at 5 level scale. The hypothesis in this study is based on Varinot, et al. [20] that fracture breakage would produce irregular shape, not rounded, and vice versa for abrasion breakage.

\subsubsection{Clemex Method}

Representative samples are spread on a glass sheet to produce a monolayer. The particles are then caught on a double graphite sticky paper that is then placed on a Scanning Electron Microscope (SEM) stub. High resolution 3D images are captured using the SEM. Images are then imported into the Clemex software, followed by running a routine that identifies the particles, and calculates their main morphology parameters (sphericity, elongation, and roughness). The values of the morphology parameters range from zero to one according to Equations (1) to (6). Roughness is evaluated based on the ratio of hull perimeter $(H P)$ to the actual particle perimeter $(P)$, as shown in Figure 4. A roughness value of one indicates a perfectly smooth particle since the hull perimeter coincides with the actual perimeter. Similarly, if the sphericity value is one, then the calculated perimeter based on the particle's surface area would be equivalent to the actual measured perimeter. For elongation, a value of one indicates an equiaxed particle since the particle's feret minimum width $(W)$ is equal to its feret maximum length $(L)$. Values close to zero, for all three parameters, suggest that the particles are rougher, less circular, and more elongated. Quantitative morphology equations are as follows [17]:

$$
\begin{gathered}
\text { Area }(A)=\frac{\pi L W}{4} \\
\text { Perimeter }(P)=\frac{\pi}{2}\left[\frac{3}{2}(L+W)-\sqrt{L W}\right] \\
\text { Aspect Ratio }(A R)=\frac{L}{W} \\
\text { Elongation }=\frac{1}{A R}
\end{gathered}
$$




$$
\begin{gathered}
\text { Sphericity }(S)=\frac{4 \pi A}{P^{2}} \\
\text { Roughness(Convexity) }=\frac{H P}{P}
\end{gathered}
$$

where: $L$ : Length, $W$ : Width, $A$ : Area. $S$ : Sphericity, $P$ : Perimeter, $H P$ : Hull Perimeter, $A R$ : Aspect Ratio. The Hull Perimeter $(H P)$ is the measure of the contour of the extrude edges of the particle as shown in Figure 5 [17].

Particles' morphology features collected from Clemex are statistically analyzed. Skewness parameter quantifies the symmetrical distribution of the property under study. Positive skewness would represent data that are leaning above the mean value, whereas negative skewness represents data leaning below the mean value. For example, in the case of abrasion breakage, the particles would be more circular, less elongated, and would exhibit smoother surfaces. The skewness values would display the following:

- Sphericity data (Sph.) would have higher negative skewness, which suggests that most of the readings are inclined towards round particles.

- Elongation (Eln.) data would be negatively skewed which indicates that most of the particles are equiaxed, rather than elongated.

- Roughness (Rgh.) data would also be negatively skewed, implying the presence of smooth surface particles.

The roughness level assessed using pre-programmed morphology software (Clemex) was not precise enough for the 3D images captured by the SEM. The software evaluated the outer contour of the particles, ignoring the $3 \mathrm{D}$ surface texture. Hence, the values were biased towards smooth particles. Therefore, the manual point count methodology was developed. Since, in this study, roughness is the main parameter to be assessed, manual point counting focused on roughness evaluation.

\subsubsection{Manual Point Counting Method}

As an alternate method to the Clemex software, manual point counting was developed. The roughness values were set to 5 levels: R1, R2, R3, R4, and R5 [21]. The roughest particle was given a value of 5 , whereas the smoothest particle was given a value of 2 . The degree of roughness increased from 2 to 5 . Roughness level 1 was given to surfaces that were round, with no sharp edges, but had hammered feature. It was speculated that an R1 particle would have been exposed to both compressive, impact and abrasive loading. Detailed definitions
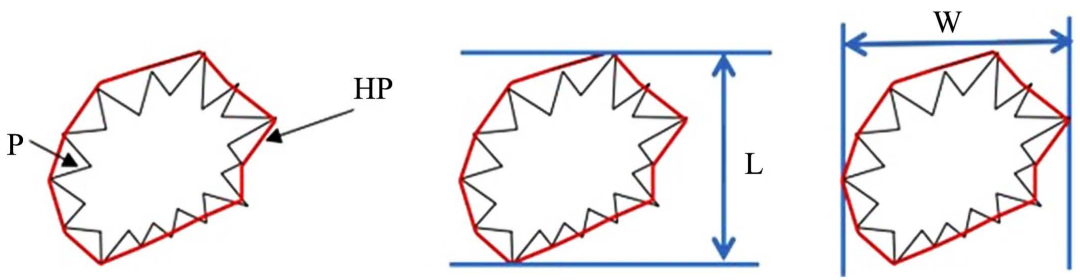

Figure 5. Particle perimeter and Hull Perimeter (HP), Length (L) and Width (W). 
and illustrations of roughness levels for quartz and galena are presented in Table 2. An excel macro subroutine was designed using visual basic to assist with the counting process. The subroutine accumulated the counts for the roughness values between 1 and 5 . The counter is trained on recognizing the roughness levels of the particles. A grid is placed on the SEM 3D-printed images to assist the counter to trace the captured images of the particles, identify and count the particles' roughness levels.

Sensitivity analysis was performed to evaluate the effect of individual counters' perspective on the results. Three different individuals were trained on the manual point counting method. The three counters were given the same sample to count, and the results were compared. The results showed that the match among the counters was very close with a maximum difference of about $6 \%$. The sensitivity analysis was verified for the three types of ground minerals and different sample size fractions, details are presented by Roufail [18].

\section{Results and Discussion}

\subsection{Clemex Results}

Detailed statistical skewness values for the morphology parameters, sphericity, elongation, and roughness are listed in Table 3. Almost all the parameters are negatively skewed. The higher the absolute skew value indicates the intensity of the skewness, which in turn indicates that the dominant type of breakage is

Table 2. Morphology roughness level definitions and illustration.

\begin{tabular}{|c|c|c|c|}
\hline \multirow{2}{*}{$\begin{array}{l}\text { Roughness } \\
\text { Level }\end{array}$} & \multirow{2}{*}{ Definition } & \multicolumn{2}{|c|}{ Illustration } \\
\hline & & Quartz & Galena \\
\hline $\begin{array}{c}\mathrm{R} 1 \\
\text { Hammered }\end{array}$ & $\begin{array}{l}\text { Round but } \\
\text { hammered } \\
\text { surface. }\end{array}$ & & \\
\hline $\begin{array}{c}\mathrm{R} 2 \\
\text { Smoothest }\end{array}$ & $\begin{array}{l}\text { Round and less } \\
\text { rough surfaces. }\end{array}$ & & \\
\hline $\begin{array}{c}\text { R3 } \\
\text { Semi-Rough }\end{array}$ & $\begin{array}{l}\text { Partially round, } \\
\text { partially angled } \\
\text { and partially } \\
\text { rough surfaces. }\end{array}$ & & \\
\hline $\begin{array}{c}\text { R4 } \\
\text { Rougher }\end{array}$ & $\begin{array}{ll}\text { - } & \text { For quartz: } \\
\text { partially round and } \\
\text { rougher surfaces. } \\
\text { - } & \text { For Galena: } \\
& \text { square edges. }\end{array}$ & & \\
\hline $\begin{array}{c}\text { R5 } \\
\text { Roughest }\end{array}$ & $\begin{array}{l}\text { Rough and sharp } \\
\text { angled surfaces. }\end{array}$ & & \\
\hline
\end{tabular}


Table 3. Clemex-morphology skewnes values.

\begin{tabular}{ccccccccc}
\hline \multirow{2}{*}{ Material } & \multicolumn{3}{c}{$1^{\text {st }}$ Pass Results } & \multicolumn{4}{c}{$4^{\text {th }} \mathbf{5}^{\text {th }}$ Pass Results } \\
\cline { 2 - 9 } & Sample & Sph. & Eln. & Rgh. & Sample & Sph. & Eln. & Rgh. \\
\hline $\begin{array}{c}\text { Galena } \\
\text { Concentrate }\end{array}$ & G1000-P1 & -0.45 & -0.77 & -1.26 & G1000-P5 & -0.69 & -0.78 & -1.53 \\
& G2000-P1 & 0.00 & -0.61 & -0.55 & G2000-P4 & -0.43 & -1.22 & -1.06 \\
Quartz & Q1000-P1 & -0.79 & -0.13 & -0.90 & Q1000-P5 & -0.18 & -0.36 & -1.49 \\
& Q2000-P1 & -0.20 & -0.61 & -2.00 & Q2000-P5 & -0.61 & -0.91 & -3.06 \\
$\begin{array}{c}\text { Mixed Quartz } \\
\text { \& Galena }\end{array}$ & M1000-P1 & -0.14 & -0.55 & -1.33 & M1000-P5 & -0.30 & -0.60 & -0.60 \\
Concentrate & M2000-P1 & -0.14 & -0.50 & -1.36 & M2000-P5 & -0.29 & -0.77 & -1.58 \\
\hline
\end{tabular}

abrasion. The samples are identified as sample type, agitator speed and number of passes through the mill. For example, G1000-P1 represents, Galena sample, tested at 1000-rpm mill agitator speed and sample collected after first pass through the mill.

Galena concentrate sample (G1000-P1) had higher negative skew values at low speed, implying that the lower agitator speed $(1000 \mathrm{rpm})$ created abrasion breakage more than the higher agitator speed (2000 rpm, G2000-P1). On the other hand, the initial breakage of quartz, data from pass 1, (Q2000-P1) showed an abrasion breakage mode at the higher agitator speed (2000 rpm), with more spherical, less elongated, and smoother particles. Whereas, the lower agitator speed, 1000 rpm, (Q1000-P1) showed fewer abrasion features, since hardly any grinding took place at the low agitator speed as indicated by the particle size distribution analysis. As for the mixed quartz and galena concentrate sample, abrasion breakage was dominant for both agitator speeds (M1000-P1, M2000-P1). This is demonstrated by similar negative skewness values for sphericity, elongation, and roughness. It is speculated that the quartz in the mixed sample behaved as a grinding media to the galena, since quartz is a harder mineral. Therefore, galena particles in the mixed sample showed abrasion features at a high agitator speed (M2000-P1), unlike the breakage trend of the galena concentrate sample at the same agitator speed (G2000-P1).

Extending residence time by increasing the number of passes of the particles through the mill promoted abrasion, which are identified as fewer rough particles with higher negative skew values for the three minerals. Quartz (Q2000-P5) showed the highest abrasion, occurring at high agitator speed and extended residence time.

Visual inspection of the particles analyzed demonstrated that there are particles that exhibit rough surfaces but was counted as smooth because Clemex identifies the outer wire frame of the particle and not the 3D surface quality, which resulted in biased analysis towards smooth particles, rather than rough. To confirm the effect of grinding operation and mineral type on particle breakage mechanism, manual point counting is designed and implemented. 


\subsection{Manual Point Counting}

Manual point counting data were analyzed using Pearson's time correlation. The same sample types used for Clemex are used for manual point counting. However, the analysis only focused on identifying the roughness levels of the particles which would predict the mechanism of particle breakage. Pearson's Equation (7) is used for analysis, which correlates mill residence time to number of particles counted at different roughness levels. Pearson's equation and variables are as follows:

$r$. Pearson's correlation, $X_{i}$ is the residence time and $Y_{i}$ is the number of particles counted for each degree of roughness and $X, Y$ are the mean values of residence time and number of particles, respectively.

$$
r=\frac{\sum_{i=1}^{n}\left(X_{i}-X\right)\left(Y_{i}-Y\right)}{\sqrt{\sum_{i=1}^{n}\left(X_{i}-X\right)^{2} \sqrt{\sum_{i=1}^{n}\left(Y_{i}-Y\right)^{2}}}}
$$

Pearson's time correlation agreed with the Clemex results according to Roufail et al. [21]. Pearson correlation coefficient indicates the association and linear strength of two variables. Correlation values range from +1 to -1 . Positive value closer to +1 indicates a strong linear positive relationship. Negative value, closer to -1 indicates an inverse relationship. A value closer to zero indicates a weak relationship.

To quantify the results, rough particles that are counted as R4 and R5, were summed, as well as the smooth particles, R1 and R2. The cumulative percent of counted rough particles $(\mathrm{R} 4+\mathrm{R} 5)$ and the smooth particles $(\mathrm{R} 1+\mathrm{R} 2)$ were plotted against the five grinding passes as shown in Figure 6 for overall trends for the 3 types of minerals. Figure 7 shows the roughness levels for different size fractions of the same mineral (quartz).

Overall trends represent combined size fraction results. Figure 6 shows the overall trend for the five passes (residence time) for galena, quartz and mixture of galena and quartz samples. Overall trend is the cumulative counts for all size fractions counted for each sample for the 5 residence times. The number of rough particles decreased, and the number of smooth particles increased as residence time in the mill increased. In other words, fracture and abrasion breakage mechanism occurrences converge as particles reside in the mill for a longer time. However, the trends showed that the percent of rough particles $(\mathrm{R} 4+\mathrm{R} 5)$ were consistently higher than the smooth particles $(\mathrm{R} 1+\mathrm{R} 2)$ at all agitator speeds. Results imply that breakage via fracture continues to occur even after relatively long residence time.

The initial breakage (P1) of the galena concentrate sample at a high agitator speed $(2000 \mathrm{rpm})$ generated similar amounts of rough particles at a low agitator speed $(1000 \mathrm{rpm})$. The galena concentrate sample generated $73 \%$ rough particles $(\mathrm{R} 4+\mathrm{R} 5)$ at a high agitator speed $(2000 \mathrm{rpm})$ and $71 \%$ at a low agitator speed (1000 rpm), Figure 6(b). Quartz, on the other hand, generated 61\% rough particles $(\mathrm{R} 4+\mathrm{R} 5)$ at a high agitator speed $(2000 \mathrm{rpm})$, compared to $78 \%$ rough 


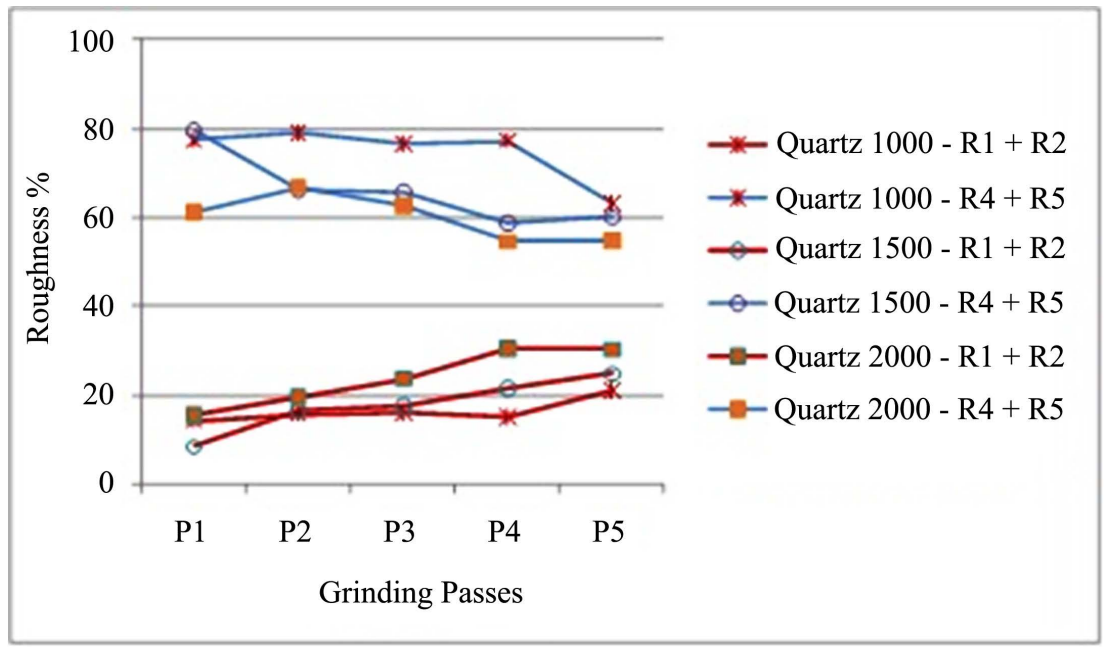

(a)

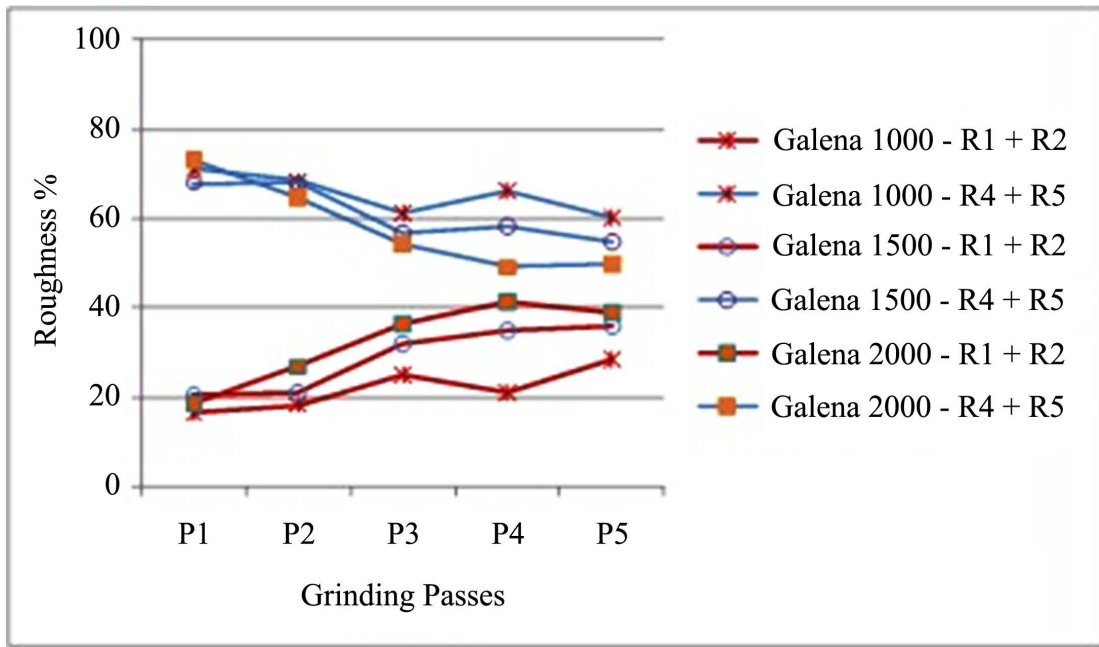

(b)

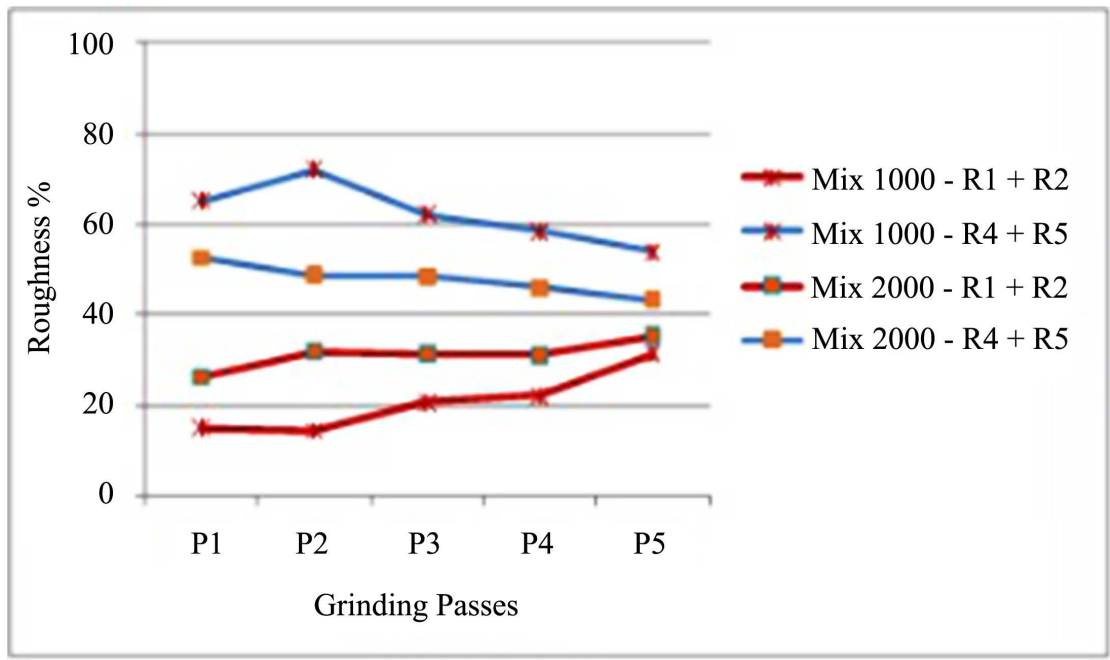

(c)

Figure 6. Overall roughness trend. (a) Quartz; (b) Galena concentrate; (c) Mixed quartz and galena concentrate. 


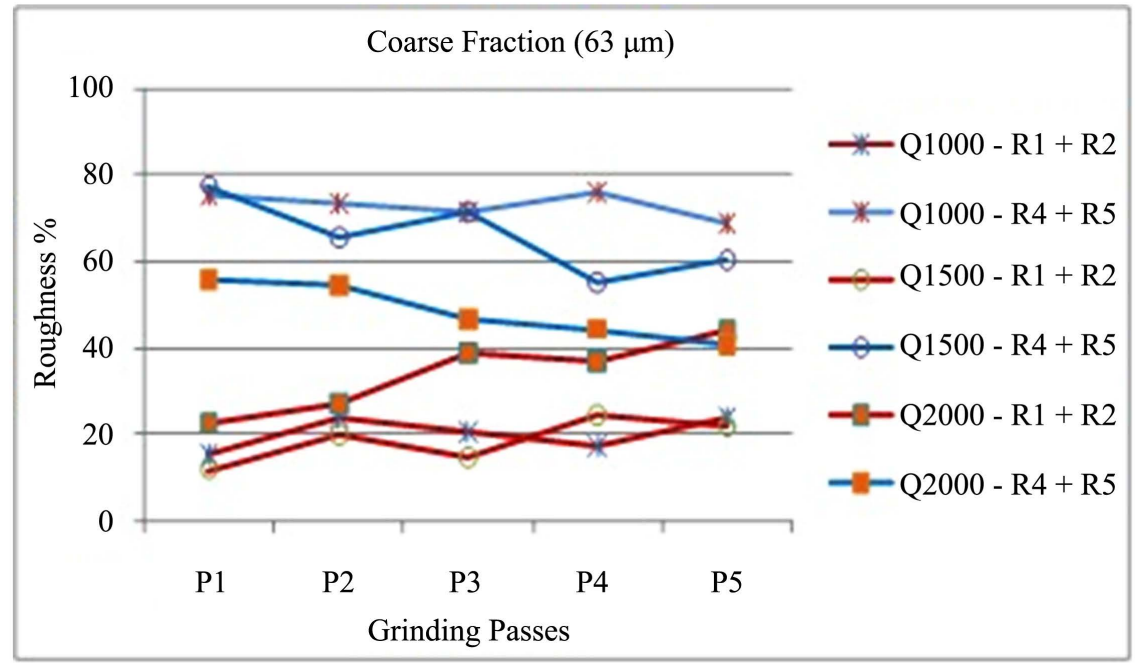

(a)

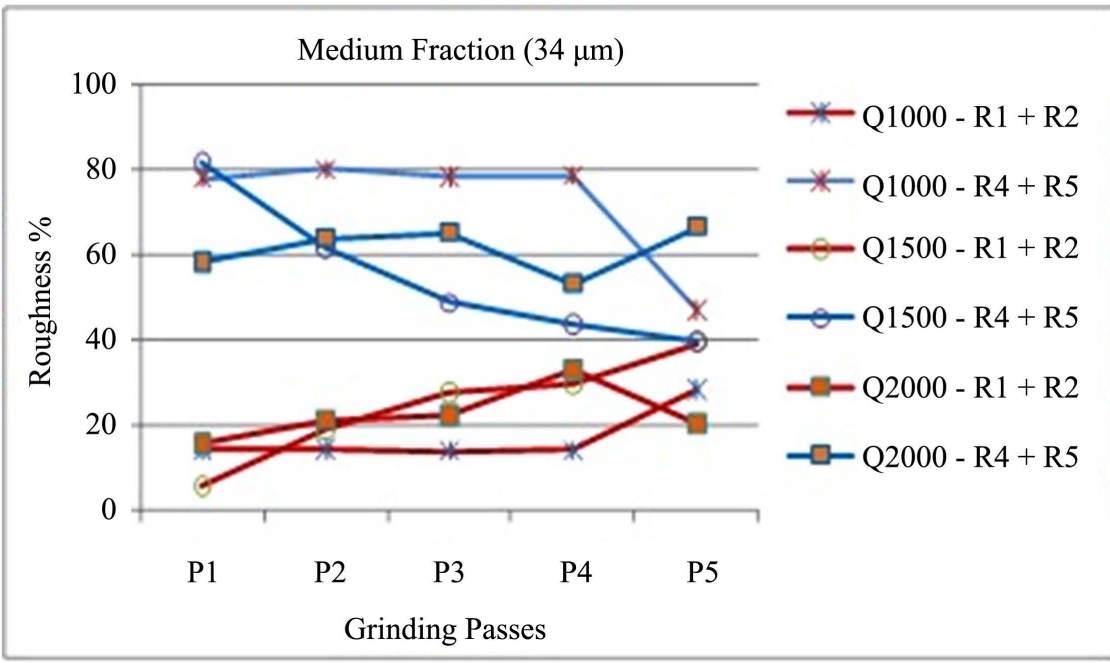

(b)

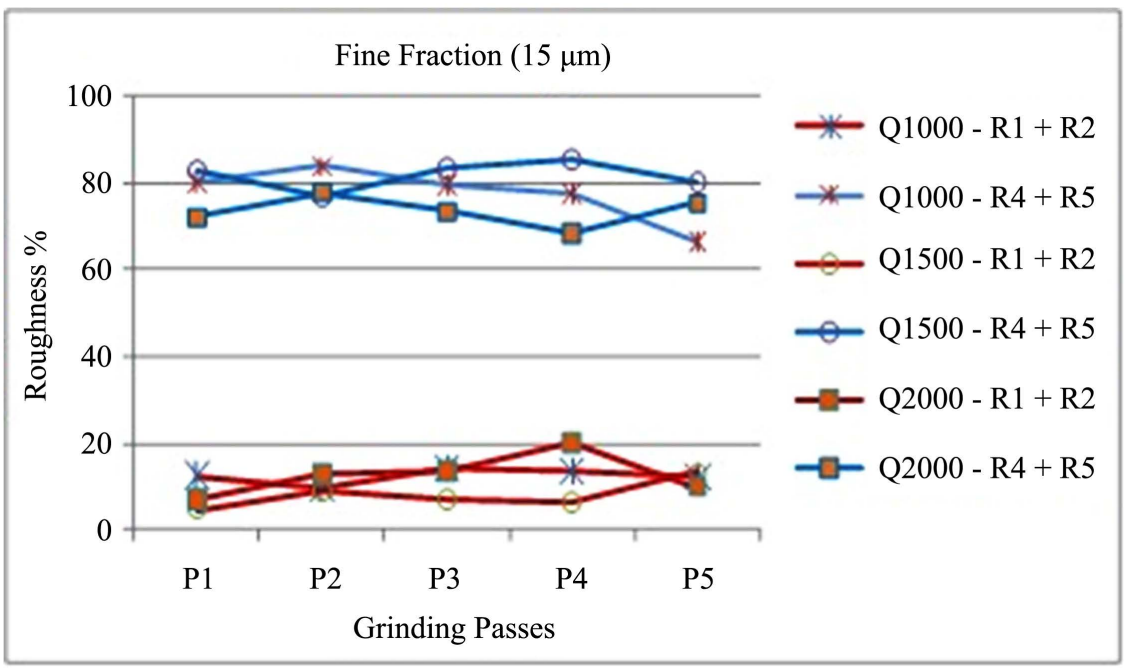

(c)

Figure 7. Roughness trend of quartz for (a) Coarse, (b) Medium \& (c) Fine fractions. 
particles (R4 + R5) at a low agitator speed (1000 rpm), Figure 6(a). Results indicated that fracture breakage mechanism, which is indicated by rough morphology, occurs for both types of minerals at high and low agitator speeds, but with different amounts. Galena concentrate had similar dominant fracture mechanism as an initial breakage (P1) for both high and low speeds. Quartz, on the other hand, had more fracture breakage mechanism at low agitator speed compared to high speed operation.

When comparing the distribution of the different roughness levels for different mineral types, quartz, and galena concentrate, at each agitator speed, the data revealed that the percent of smooth particles of galena concentrate (R1 + R2) are increasing and percent of rough particles $(\mathrm{R} 4+\mathrm{R} 5)$ are declining at higher rate than that for quartz, respectively. This relationship implies that galena, soft, heavy mineral, would experience abrasion breakage by increasing residence time, compared to quartz, which is harder, lighter mineral.

To understand the distribution of roughness at different size fractions, the data for three size fractions were plotted as percent of rough $(\mathrm{R} 4+\mathrm{R} 5)$ and smooth $(\mathrm{R} 1+\mathrm{R} 2)$ particles against residence time (number of passes). The plots were prepared for the three minerals tested, quartz, galena, mixed quartz-galena samples. The trend lines for the three minerals were similar, therefore, in this paper only quartz results are presented as sample data, Figure 7 . The three size fractions (coarse, medium, and fine) for quartz had geometric mean sizes of 63 $\mu \mathrm{m}, 34 \mu \mathrm{m}$ and $15 \mu \mathrm{m}$. The trend line showed that for the coarse and medium size fraction, Figure 7(a) and Figure 7(b), percent of rough particles decreased per pass (increase of residence time) and smooth particles increased. On the other hand, the fine fraction, Figure $7(\mathrm{c})$, trend line was flat throughout the five passes. The rough particles were about $65 \%-80 \%$ of the total particles counted, whereas the smooth particles were about $10 \%-20 \%$. Coarse and medium fractions, initially brake via fracture, producing relatively a high number of rough particles. As grinding residence time increases, abrasion breakage mode becomes more evident, where the count of smooth particles increases. This observation also indicates that the finer products, which should contain a significant amount of progeny particles from the coarser fractions, were consistently broken via fracture with no significant effect of time on the mode of breakage.

\section{Conclusions}

Stirred mill products are analyzed for their morphological features using quantitatively automated (Clemex) and manual point counting methods. The particles were ground to fine size using a horizontal high-speed stirred mill. The parameters studied were the stress intensity and type of mineral on the breakage mechanism. The stress intensity is evaluated by changing the agitator speed of the mill and the type of mineral is studied by choosing two types of minerals with extreme physical properties, which were galena and quartz. The breakage effect of the minerals on each other is also addressed by creating a mix of both types of 
minerals galena and quartz with a ratio of 1:6 by volume. Morphological parameters considered were the roundness, elongation, and surface roughness of the particles. However, roughness is the main parameter that would indicate the type of particle breakages such as fracture or abrasion. Rough particles imply fracture breakage, along grain boundaries (intergranular), while smooth particles imply abrasion breakage, across the grains (transgranular). Operating conditions would favor the breakage mechanism, which in turn will control the produced shape of the particles.

The following conclusions were drawn from this study:

- Results validate that the initial particle breakage mechanisms in stirred mills are dominantly fractured. As grinding residence time increases, attrition and abrasion breakage mechanism becomes more evident.

- Mineral types show different breakage mechanisms at extreme agitator speeds. Galena concentrate had similar dominant fracture breakage along the grain boundaries at the initial breakage stage, at both high and low agitator speeds. Quartz, on the other hand, breaks via fracture at low agitator speed while abrading at higher speed. The breakage mechanism of selected minerals can be controlled by manipulating mill operation.

- The overall trend demonstrated that the number of rough particles was consistently higher than smooth particles. Rough particles suggest that breakage occurs along grain boundaries and fracture is the dominant breakage mechanism, which is contradicting the common notion that fine grinding breaks particles mostly via abrasion.

- Increasing grinding residence time, abrasion breakage mechanism becomes dominant, which leads to more fine ground product. Fine products complicate downstream mineral processing operations. Therefore, avoiding long residence time and controlling mill operation parameters that achieve targeted mineral liberation at the coarsest possible product size is recommended.

\section{Acknowledgements}

This work is a partial product of research performed under the supervision of Dr. Bern Klein, Dr. Peter Radziszewski and Dr. Andy Stradling. Their valuable advice and support are highly appreciated. The technical support of Teck Cominco Ltd., ART and G\&T Metallurgical Services Ltd. is much appreciated.

\section{Conflicts of Interest}

The authors declare no conflicts of interest regarding the publication of this paper.

\section{References}

[1] Singh, V., Dixit, P., Venugopal, R. and Venkatesh, B. (2018) Ore Pretreatment Methods for Grinding: Journey and Prospects. Mineral Processing and Extractive Metallurgy Review, 40, 1-15. https://doi.org/10.1080/08827508.2018.1479697 
[2] Becker, M., Kwade, A. and Schwedes, J. (2001) Stress Intensity in Stirred Media Mills and Its Effect on Specific Energy Requirement. International Journal of Mineral Processing, 61, 189-208. https://doi.org/10.1016/S0301-7516(00)00037-5

[3] Becker, M. and Schwedes, J. (1999) Comminution of Ceramics in Stirred Media mills and Wear of Grinding Beads. Powder Technology, 105, 374-381. https://doi.org/10.1016/S0032-5910(99)00161-8

[4] Kwade, A. and Schwedes, J. (2002) Breaking Characteristics of Different Materials and Their Effect on Stress Intensity and Stress Number in Stirred Media Mills. Powder Technology, 122, 109-121. https://doi.org/10.1016/S0032-5910(01)00406-5

[5] Blecher, L., Kwade, A. and Schwedes, J. (1996) Motion and Stress Intensity of Grinding Beads in a Stirred Media Mill. Part 1: Energy Density Distribution and Motion of Single Grinding Beads. Powder Technology, 86, 59-68. https://doi.org/10.1016/0032-5910(95)03038-7

[6] Partyka, T. and Yan, D. (2007) Fine Grinding in a Horizontal Ball Mill. Minerals Engineering, 20, 320-326. https://doi.org/10.1016/j.mineng.2006.12.003

[7] Peukert, W. (2004) Material Properties in Fine Grinding. International Journal of Mineral Processing, 74, S3-S17. https://doi.org/10.1016/j.minpro.2004.08.006

[8] Bu, X., Chen, Y., Ma, G., Sun, Y., Ni, C. and Xie, G. (2019) Differences in Dry and Wet Grinding with a High Solid Concentration of Coking Coal Using a Laboratory Conical Ball Mill: Breakage Rate, Morphological Characterization, and Induction Time. Advanced Powder Technology, 30, 2703-2711. https://doi.org/10.1016/j.apt.2019.08.016

[9] Moosakazemi, F., Tavakoli Mohammadi, M.R., Mohseni, M., Karamoozian, M. and Zakeri, M. (2017) Effect of Design and Operational Parameters on Particle Morphology in Ball Mills. International Journal of Mineral Processing, 165, 41-49. https://doi.org/10.1016/j.minpro.2017.06.001

[10] Tromans, D. and Meech, J.A. (2002) Fracture Toughness and Surface Energies of Minerals: Theoretical Estimates for Oxides, Sulphides, Silicates and Halides. Minerals Engineering, 15, 1027-1041. https://doi.org/10.1016/S0892-6875(02)00213-3

[11] Celik, I.B. and Oner, M. (2006) The Influence of Grinding Mechanism on the Liberation Characteristics of Clinker Minerals. Cement and Concrete Research, 36, 422-427. https://doi.org/10.1016/j.cemconres.2005.09.011

[12] Alex, T.C., Kumar, R., Roy, S.K. and Mehrotra, S.P. (2008) Stirred Bead Mill Grinding of Gibbsite: Surface and Morphological Changes. Advanced Powder Technology, 19, 483-491. https://doi.org/10.1016/S0921-8831(08)60914-0

[13] Yang, H., Liu, F. and Pan, X. (2018) Mineral Structure and Crystal Morphologies of High-Iron Hydrargillite. International Journal of Minerals, Metallurgy, and Materials, 25, 505-514. https://doi.org/10.1007/s12613-018-1597-4

[14] Yang, B., Wu, A., Narsilio, G.A., Miao, X. and Wu, S. (2017) Use of High-Resolution X-Ray Computed Tomography and 3D Image Analysis to Quantify Mineral Dissemination and Pore Space in Oxide Copper Ore Particles. International Journal of Minerals, Metallurgy, and Materials, 24, 965-973.

https://doi.org/10.1007/s12613-017-1484-4

[15] Lecoq, O., Guigon, P. and Pons, M.N. (1999) A Grindability Test to Study the Influence of Material Processing on Impact Behaviour. Powder Technology, 105, 21-29. https://doi.org/10.1016/S0032-5910(99)00114-X

[16] Donskoi, E., et al. (2007) Utilization of Optical Image Analysis and Automatic Texture Classification for Iron Ore Particle Characterisation. Minerals Engineering, 20, 461-471. https://doi.org/10.1016/j.mineng.2006.12.005 
[17] User Guide, Vision PE \& Vision Lite 6.0. Clemex Technologies Inc., 2009.

[18] Reem, A.R. (2011) The Effect of Stirred Mill Operation on Particles Breakage Mechanism and Their Morphological Features. University of British Columbia, Vancouver.

[19] Parry, J.M. (2006) Ultrafine Grinding for Improved Mineral Liberation in Flotation Concentrates. https://open.library.ubc.ca/collections/831/items/1.0081153

[20] Varinot, C., Hiltgun, S., Pons, M.N. and Dodds, J. (1997) Identification of the Fragmentation Mechanisms in Wet-Phase Fine Grinding in a Stirred Bead Mill. Chemical Engineering Science, 52, 3605-3612. https://doi.org/10.1016/S0009-2509(97)89693-5

[21] Roufail, R., Klein, B. and Blaskovich, R. (2011) Effect of Grinding Operation on Product Morphology in Stirred Mills. Proceedings of the Canadian Mineral Processors Conference, Ottawa, 18-20 January 2011.

https://www.onemine.org/document/abstract.cfm?docid=232377 